\title{
Generic study of solar radiation and solar wind sailing
}

\begin{abstract}
With the progress of solar sail interplanetary travel in the last two decades, various concepts and technologies have been developed. To that end, two means of sailing, Solar Sail and Electric Sail, will be discussed. The capabilities as well as performance aspects of both technologies will be compared by studying different generic missions such as escape orbit and local optimizations of orbital parameters.
\end{abstract}

Keyword: Electric sail; Interplanetary travel; Orbital dynamics; Propellantless propulsion; Solar radiation pressure; Solar sailing 